\title{
The effect of PMSG dose on the incidence of chromosomal anomalies in mouse embryos fertilized in vitro
}

\author{
I. Maudlin* and Lynn R. Fraser*† \\ *Clinical Research Centre, Watford Road, Harrow, Middlesex HAI 3 UJ and \\ $\uparrow$ Department of Obstetrics and Gynaecology, University College Hospital, \\ London WCIE 6DH, U.K.
}

\begin{abstract}
Summary. Chromosomal analysis of first-cleavage mouse embryos obtained by fertilization of TO eggs with TO spermatozoa in vitro revealed that the incidence of polyploidy was proportional to the dose of PMSG given to the donor females. The doseresponse was shown to be linear, with the level of polyploidy rising from $8.0 \%$ with a PMSG dose of 1.5 i.u./female to $20.8 \%$ with 10.0 i.u./female. A total of 64 polyploid embryos was found among the 452 examined and in all cases the polyploidy was the result of polyspermy or fertilization by diploid spermatozoa.
\end{abstract}

\section{Introduction}

The induction of ovulation in mammals by administration of exogenous gonadotrophin may be responsible for an increased incidence of chromosomal abnormalities in the resulting embryos. Fujimoto, Pahlavan \& Dukelow (1974) and Fujimoto, Passantino \& Koenczoel (1975) noted slight, but statistically insignificant, increases in chromosomal abnormalities in rabbit blastocysts and intratubal embryos obtained after superovulation, when compared with a control group. Boué \& Boué (1973) found a significant increase in the level of trisomy among abortuses from women who had been induced to ovulate when compared with a sample of untreated women.

Experiments with mouse embryos in vivo have shown that a low level of chromosomal anomalies normally occurs. In studies of first-cleavage mouse embryos, Donahue (1972a) found an incidence of $1.8 \%$ aneuploidy and $1.5 \%$ polyploidy, while Kaufman (1973) found $8.3 \%$ to be aneuploid and $4.1 \%$ polyploid. We found a significantly greater incidence of triploidy in mouse morulae fertilized in vitro $(12.8 \%)$ than in those fertilized in vivo $(2.7 \%)$ (Fraser, Zanellotti, Paton \& Drury, 1976), and this in-vitro fertilization technique was therefore adopted to study the effects of gonadotrophin dosage on chromosomal anomalies in the mouse. However, because a rather low proportion (33\%) of analysable embryos was obtained from morulae (Fraser et al., 1976), in the present study we used firstcleavage embryos which yield a high proportion of scoreable preparations. Only the PMSG dose was varied because the work of Shaver (1970) had indicated that variation in HCG dosage apparently did not affect the chromosome constitution of rabbit embryos.

\section{Materials and Methods}

\section{Fertilization in vitro}

Outbred TO mice, 2-4 months old, were obtained from the Specific Pathogen Free breeding unit of the Clinical Research Centre. Virgin females were induced to ovulate by an i.p. injection of PMSG (Gestyl : Organon) followed $48 \mathrm{~h}$ later by $5.0 \mathrm{i} . \mathrm{u}$. HCG (Pregnyl: Organon). The PMSG was diluted in distilled water so that animals received $1 \cdot 5,4 \cdot 5,7 \cdot 5$ or $10 \cdot 0 \mathrm{i}$.u. in a volume of $0 \cdot 1 \mathrm{ml}$ and at least two different levels of PMSG stimulation were examined in each experiment.

The methods and media used for fertilization in vitro were as described by Fraser \& Drury (1975). After a 20-min dispersal period, epididymal sperm suspensions were diluted to a concentration of $2 \times 10^{6}$ spermatozoa/ml. Unfertilized eggs, obtained $13 \mathrm{~h}$ after the HCG injection, were released 
directly into the sperm suspensions and incubated at $37^{\circ} \mathrm{C}$ in an atmosphere of $5 \% \mathrm{O}_{2}, 5 \% \mathrm{CO}_{2}$, $90 \% \mathrm{~N}_{2}$ for $6 \mathrm{~h}$. Eggs were then washed twice in culture medium containing $4 \mathrm{mg}$ bovine serum albumin (Armour)/ml and transferred to droplets of culture medium containing $10^{-5} \mathrm{~mm}$-vinblastine sulphate (Velbe: Lilly). After overnight culture in this medium, the eggs were fixed and stained according to the method of Tarkowski (1966).

Initially, all eggs (i.e. with and without a second polar body) were cultured in Velbe-containing medium. However, because those eggs lacking a second polar body proved to be unfertilized when examined after fixation, in most experiments only eggs possessing a polar body were analysed. To verify that this did not select against potential digynic triploids which would not have had second polar bodies, the eggs which appeared to be unfertilized were cultured in Velbe-free medium. Such eggs were never observed to cleave and because experimentally produced digynic triploid TO embryos cleave normally (L. R. Fraser, unpublished observation), it was presumed that this class of embryos was absent from this study.

\section{Fertilization in vivo}

Virgin TO females, 2-4 months old, were injected i.p. with either a low dose (1.5 i.u.) or a high dose (7.5 i.u.) of PMSG and, approximately $48 \mathrm{~h}$ later, with a dose of 5.0 i.u. HCG. The HCG was injected 12-13 $\mathrm{h}$ before the estimated time of natural ovulation and the females were paired with TO males. Females which had a vaginal plug the following day were killed in the afternoon and 1-cell, fertilized eggs were recovered from the oviducts. When necessary, eggs were transferred to culture medium containing $2 \mathrm{mg}$ hyaluronidase (bovine testes, Type I: Sigma)/ml to remove cumulus cells still attached to the zona pellucida. All eggs were washed twice, transferred to droplets of culture medium containing $10^{-5} \mathrm{~mm}$-Velbe, cultured overnight and then fixed and stained as above.

\section{Analysis of preparations}

Because a high incidence of polyploidy was obtained (see 'Results'), attempts were made to determine whether this was due to supernumerary male or female nuclei. Preliminary experiments were carried out using exactly the same methods but with males having a T6 marker chromosome to distinguish the male complement. It was clear from the analysis of 504 spreads that the sperm-derived chromosomes were less condensed than those derived from the egg (PI. 1, Fig. 1). This differential condensation was also apparent in the present study and has previously been noted by McGaughey \& Chang (1971) and Donahue (1972a).

\section{Results}

\section{Polyploidy}

Polyploidy was the more frequently detected anomaly in this study. Of the 536 embryos examined after in-vitro fertilization, 452 yielded scoreable preparations $(84 \%) ; 64$ were polyploid $(14.2 \%)$ and of these 60 were triploid (Pl. 1, Fig. 2; Pl. 2, Fig. 4) and 4 were tetraploid (Pl. 2, Fig. 3). In comparison, 4 out of $228(1.8 \%)$ embryos fertilized in vivo were found to be triploid. The results are presented in Table 1.

The control data from individual experiments were tested for heterogeneity and were shown to be homogeneous within hormone doses; these data have therefore been pooled for analysis. A $\chi^{2}$ test for heterogeneity also showed that the proportions of polyploid embryos were homogeneous within the two in-vitro groups of 1.5 and 7.5 i.u. PMSG. A straightforward comparison was therefore made between these in-vivo and in-vitro group totals; there was no significant difference between the two groups at 1.5 i.u., but at 7.5 i.u. there was a highly significant difference $\left(\chi^{2}=21 \cdot 5, P<0 \cdot 001\right)$. This result with TO eggs and TO spermatozoa confirms that obtained with morulae from $(\mathrm{C} 57 \mathrm{BL} / 10 \times$ CBA)F $F_{1}$ eggs and TO spermatozoa (Fraser et al., 1976).

The proportion of polyploid embryos increased as the dose of PMSG increased. Because a simple regression analysis of these proportions was not statistically sound due to the variation between 

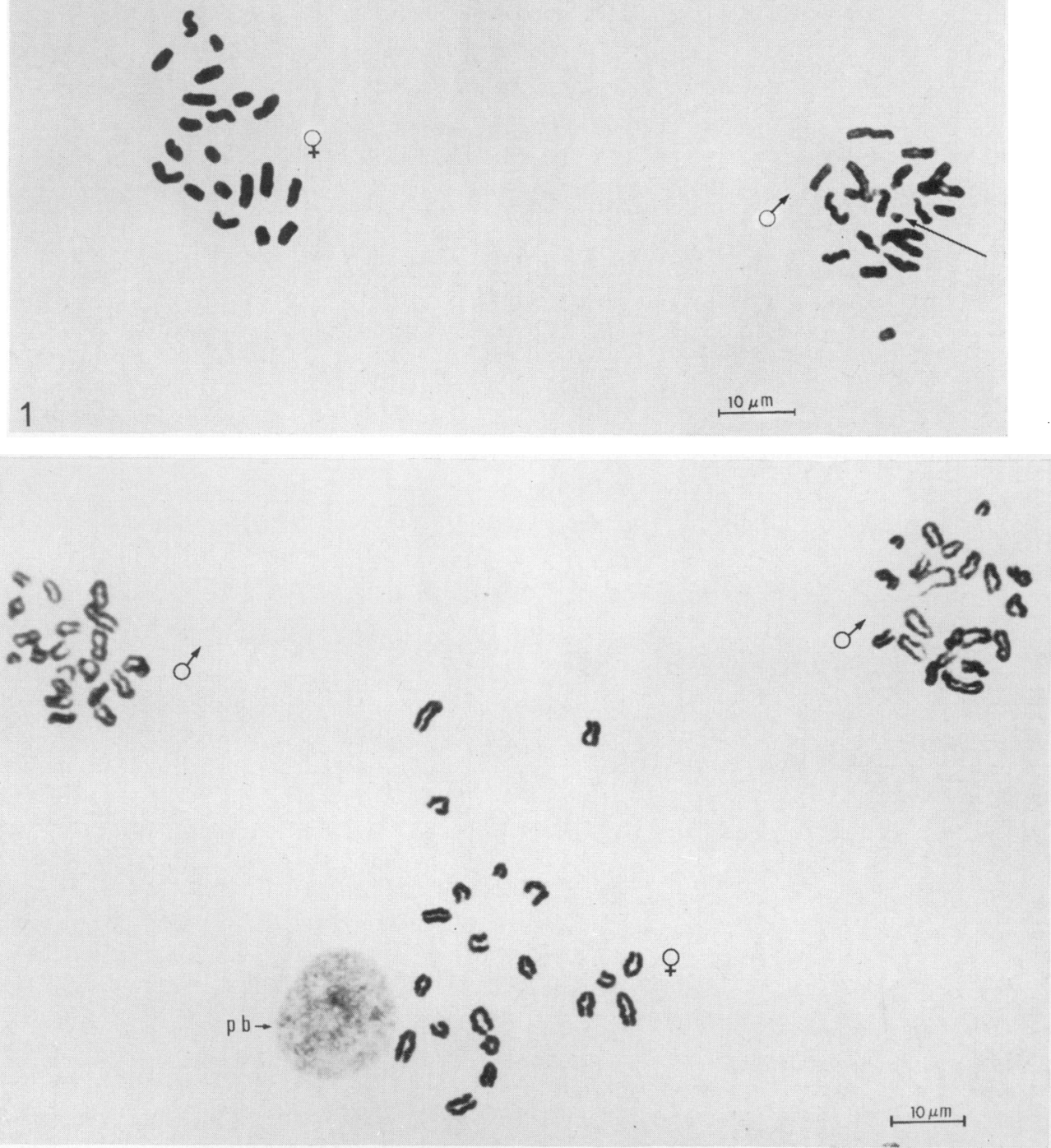

Fig. 1. First-cleavage diploid mouse embryo fertilized in vitro showing differential condensation of male and female chromosomes; the T6 marker chromosome is indicated by the arrow. $\delta=$ male complement; $q=$ female complement $; \mathrm{pb}=$ second polar body.

Fig. 2. First-cleavage mouse embryo fertilized in vitro showing a triploid chromosome complement produced by dispermy. 

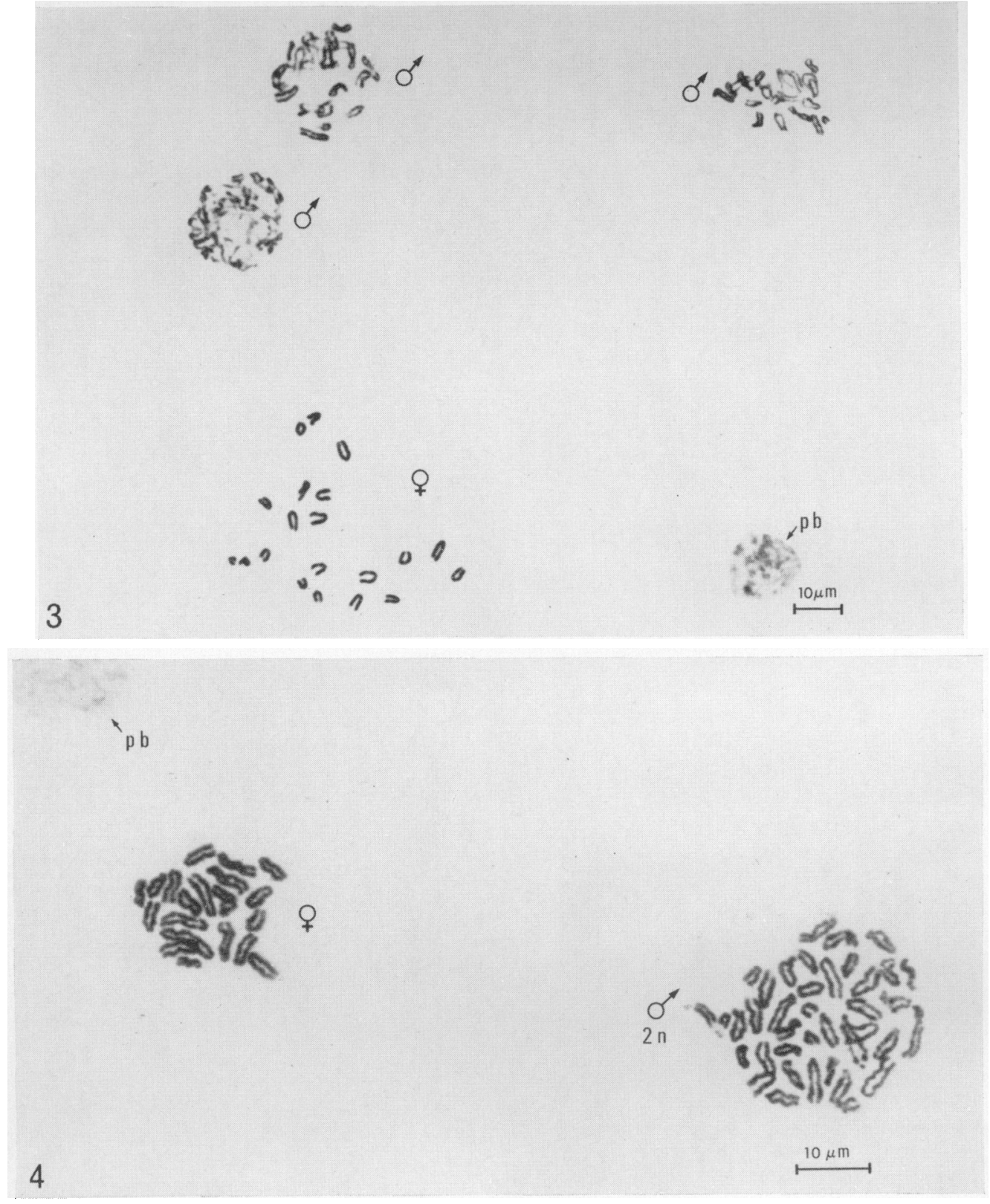

Fig. 3. First-cleavage mouse embryo fertilized in vitro showing a tetraploid chromosome complement produced by trispermy. Note the differential condensation of the three male complements, compared with that of the female chromosomes.

Fig. 4. First-cleavage mouse embryo fertilized in vitro showing a triploid chromosome complement produced by a diploid spermatozoon. 
Table 1. Incidence of polyploidy in first-cleavage mouse embryos of the TO strain, fertilized in vitro and in vivo, after administration of different doses of PMSG to donor females

\begin{tabular}{|c|c|c|c|c|c|c|c|c|c|c|c|c|}
\hline & \multicolumn{12}{|c|}{ PMSG dosage (i.u.) } \\
\hline & \multicolumn{3}{|c|}{$1 \cdot 5$} & \multicolumn{3}{|c|}{$4 \cdot 5$} & \multicolumn{3}{|c|}{$7 \cdot 5$} & \multicolumn{3}{|c|}{$10 \cdot 0$} \\
\hline & $\mathbf{N}$ & $\mathbf{n}$ & $\%$ & $\mathbf{N}$ & $\mathrm{n}$ & $\%$ & $\mathbf{N}$ & $\mathbf{n}$ & $\%$ & $\mathbf{N}$ & $\mathbf{n}$ & $\%$ \\
\hline \multicolumn{13}{|l|}{ In vitro } \\
\hline 1 & 41 & 2 & 4.9 & & & & 36 & 3 & $8 \cdot 3$ & & & \\
\hline 2 & 24 & 1 & $4 \cdot 2$ & & & & 18 & 3 & $16 \cdot 6$ & & & \\
\hline 3 & 15 & 1 & $6 \cdot 6$ & 25 & 1 & $4 \cdot 0$ & 33 & 6 & $18 \cdot 2$ & & & \\
\hline 4 & & & & 22 & 1 & $4 \cdot 5$ & 18 & 4 & $22 \cdot 2$ & & & \\
\hline 5 & 15 & 2 & $13 \cdot 3$ & 11 & 1 & $9 \cdot 1$ & 14 & 2 & $14 \cdot 3$ & 10 & 3 & 30.0 \\
\hline 6 & & & & 28 & 12 & $42 \cdot 8$ & & & & 6 & 3 & 50.0 \\
\hline 7 & & & & 12 & 1 & $8 \cdot 3$ & 16 & 2 & $12 \cdot 5$ & 18 & 1 & $5 \cdot 5$ \\
\hline 8 & 18 & 3 & $16 \cdot 7$ & 26 & 2 & $7 \cdot 7$ & 8 & 2 & $25 \cdot 0$ & 38 & 8 & $21 \cdot 1$ \\
\hline Totals & 113 & 9 & $8 \cdot 0$ & 124 & 18 & $14 \cdot 5$ & 143 & 22 & $15 \cdot 4$ & 72 & 15 & $20 \cdot 8$ \\
\hline
\end{tabular}

In vivo

$\begin{array}{lllllll}\text { Totals } & 61 & 3 & 4.9 & 167 & 1 & 0.6\end{array}$

$\mathrm{N}=$ No. of embryos examined; $\mathrm{n}=$ no. of $3 n$ embryos.

experiments within dosage groups, a series of dose-response curves were fitted by computer to each of the 8 experimental groups, using transformed data. Doses were transformed to $\log _{10}$ and the proportions of polyploid embryos were weighted to allow for the differing numbers in each proportion, a logistic transformation being applied to normalize the binomial data (Cox, 1970). A test for parallelism showed that the slopes of the lines did not differ significantly $\left(\chi^{2}=8 \cdot 7\right.$, d.f. 13$)$. The response curves had a common slope of $1 \cdot 1762 \pm 0.5722$, indicating a significant positive regression $(P=0.05-0.01)$. It was therefore concluded that there was a significant response in terms of incidence of polyploidy related to PMSG dose.

Analysis of the polyploid embryos by distinction of the paternal and maternal chromosomes (see 'Methods') showed that all 64 were produced by di- or triandry. Most of the polyploids resulted from dispermy but 4 were due to fertilization by spermatozoa with a diploid complement (Pl. 2, Fig. 4); 3 of these embryos were $3 n$ while the fourth was $4 n$ with both haploid and diploid male chromosome complements.

\section{Aneuploidy}

Aneuploid counts were designated as 'confident' or 'uncertain' depending on the quality of the chromosome spreads produced. Great care was taken in the designation of hypodiploid embryos and all hypodiploids with an irregular conformation were classified as 'uncertain'.

Table 2. Incidence of aneuploidy in first-cleavage mouse embryos of the TO strain fertilized in vitro and in vivo

\begin{tabular}{|c|c|c|c|c|c|c|c|c|c|}
\hline & \multicolumn{3}{|c|}{ Monosomic } & \multicolumn{3}{|c|}{ Trisomic } & \multicolumn{3}{|c|}{ Total aneuploid } \\
\hline & $\mathbf{C}$ & $\mathbf{U}$ & $\mathrm{C}+\mathrm{U}$ & C & $\mathbf{U}$ & $\mathbf{C}+\mathbf{U}$ & C & $\mathbf{U}$ & $\mathrm{C}+\mathrm{U}$ \\
\hline In vitro & $\begin{array}{c}7 \\
(1 \cdot 1 \%)\end{array}$ & 3 & $\begin{array}{c}10 \\
(1 \cdot 2 \%)\end{array}$ & $\begin{array}{c}6 \\
(0.9 \%)\end{array}$ & 0 & $\begin{array}{c}6 \\
(0 \cdot 7 \%)\end{array}$ & $\begin{array}{c}13 \\
(2 \cdot 0 \%)\end{array}$ & 3 & $\begin{array}{c}16 \\
(1.9 \%)\end{array}$ \\
\hline In vivo & $\begin{array}{c}1 \\
(0.3 \%)\end{array}$ & 0 & $\begin{array}{c}1 \\
(0.2 \%)\end{array}$ & $\begin{array}{c}2 \\
(0.6 \%)\end{array}$ & 0 & $\begin{array}{c}2 \\
(0.5 \%)\end{array}$ & $\begin{array}{c}3 \\
(1 \cdot 0 \%)\end{array}$ & 0 & $\begin{array}{c}3 \\
(0.7 \%)\end{array}$ \\
\hline
\end{tabular}

$C=$ 'confident' counts; 655 in vitro, 311 in vivo.

$\mathrm{U}=$ 'uncertain' counts; 198 in vitro, 118 in vivo.

The counts from male and female nuclei were combined. 
We were unable to detect any significant difference between the levels of male and female aneuploidy and the data from the two groups were therefore combined. The results in Table 2 do not show the variation among PMSG groups in the levels of aneuploidy because prior analysis showed that there was no significant effect of PMSG dose. There was clearly a greater degree of aneuploidy in the in-vitro embryos than in the in-vivo controls, but in no case was the difference statistically significant. There was no significant difference between the numbers of monosomic and trisomic aneuploids detected although there was a slight excess of monosomics in the in-vitro group, presumably due to the number of 'uncertain' counts.

\section{Discussion}

There have been conflicting reports about the effects of gonadotrophins on the embryonic development of mammals. Shaver (1970) found no indication that excess HCG given before mating adversely affected the chromosome constitution of rabbit blastocysts, and Fechheimer \& Beatty (1974) concluded that superovulation had no demonstrable effect on the incidence of heteroploidy in rabbit blastocysts. Spindle \& Goldstein (1975) found that variation of the PMSG and HCG dosage did not affect blastocyst formation or subsequent growth of mouse embryos fertilized in vivo and cultured in vitro. Conversely, Elbling (1973) reported that gonadotrophin treatment resulted in significantly increased numbers of deformed mouse fetuses, although these findings have been disputed (Smith \& Chrisman, 1975). Beaumont \& Smith (1975) found that superovulation caused substantial pre- and post-implantation losses in vivo in superovulated mice when compared to an unstimulated group.

The overall level of polyploidy recorded here for TO eggs fertilized in vitro with TO spermatozoa was $14.2 \%$, a value similar to that of $12.8 \%$ found for morulae obtained from (C57BL/10 $\times$ CBA) $F_{1}$ eggs fertilized in vitro with TO spermatozoa (Fraser et al., 1976). The present work, however, has demonstrated a simple dose-response relationship between the PMSG dose and the incidence of polyploidy, mainly triploidy, detected in TO mouse eggs fertilized in vitro. Although these results were obtained using an artificial method of fertilization, they serve to confirm the indications given by Boué \& Boué $(1973)$ and Fujimoto et al. $(1974,1975)$ that chromosomal anomalies may be induced by superovulation.

The polyploid embryos in our in-vitro study were all polyandric. In a review of the origins of polyploidy, Beatty (1970) stated that the most important form of vertebrate polyploidy was produced by digyny, but much of his evidence related to the 'silver 'strain of mice which has a high incidence of spontaneous second polar body suppression (Braden, 1957). Beatty (1974) also calculated that dispermy did not contribute at all to the incidence of polyploidy in man. Donahue (1972a, b), after studies of 1-cell mouse embryos fertilized in vivo, concluded that spontaneous triploidy was produced equally by dispermy and digyny. Wróblewska (1971) examined post-implantation mouse embryos from the cross CBA $\times$ CBA/T6T6 and found that 12 out of 18 triploids were apparently digynic in origin while the remaining 6 could not be assessed. Similarly, Fechheimer \& Beatty (1974) in a study of rabbit blastocysts concluded that, although dispermy could not be ruled out, digyny was the simplest explanation for triploidy. Takagi \& Sasaki (1976), who studied A/He mouse embryos fertilized in vivo after superovulation, found that most of the triploids were digynic. The incidence of triploidy $(4.5 \%)$ detected by Takagi \& Sasaki (1976) in the absence of hormonal stimulation was similar to that found in the 'silver' strain (Beatty, 1970), suggesting that the A/He strain, like the 'silver' strain, is prone to failure of the second meiotic division and therefore to the increased production of digynic embryos. Despite this apparent genetic predisposition to digyny, Takagi \& Sasaki (1976) still found $1 \cdot 7-4.5 \%$ of the zygotes examined to be diandric in origin which corresponds well with the $0.6-4.9 \%$ triploid and diandric embryos obtained in vivo in the present work. Comparison with our work is complicated by the in-vitro technique employed here, but this should not have inhibited the formation of digynic embryos. Indeed, the 4 triploid embryos in the in-vivo groups were all diandric in origin, while the incidence of triploidy $(1.8 \%)$ was very close to the $1.2 \%$ recorded by Donahue (1972a).

The response to PMSG demonstrated here by an increase in polyploidy and the polyandric nature of this polyploidy suggests that the hormone affects extra-nuclear events rather than the egg nucleus. 
This might involve changes in the properties of the zona pellucida to permit entry of more than one spermatozoon or in the vitelline block to polyspermy which would normally allow only one spermatozoon to fuse with the egg (Austin, 1961). Morphological (Katzberg \& Henrickx, 1966) and biochemical (Conrad, Buckley \& Stambaugh, 1971; Fraser \& Dandekar, 1975) changes have been noted in the zonae pellucidae of mammalian eggs obtained by superovulation. It is evident that the block to polyspermy, whether at the zona pellucida or the vitelline membrane, is less effective in vitro than in vivo, perhaps reffecting the inability of the egg to respond adequately in the presence of relatively high numbers of spermatozoa in vitro. Nevertheless, even though all the eggs in our study were mixed with the same concentration of spermatozoa, those eggs stimulated by lower doses of PMSG were more resistant to penetration by extra spermatozoa than those obtained with high doses of hormone.

The presence of diploid spermatozoa in 4 of the embryos examined (i.e. $1 \%$ ) raises interesting questions as to the frequency of their occurrence in vivo. In a microdensitometric study, Carothers \& Beatty (1975) found only 1 diploid spermatozoon outside their randomly chosen sample of 1000 mouse spermatozoa obtained from the 'silver' strain, specifically selected because of its high incidence of polyploidy. However, the 'silver' strain is noted for a high incidence of triploidy, mainly due to suppression of the second polar body in the egg (Braden, 1957). Stolla \& Gropp (1974) also failed to find diploid spermatozoa in $M$. musculus and $M$. poschiavinus but did find a low incidence in the $F_{1}$ hybrid. There is, however, ample evidence from other mammals to show that diploid spermatozoa do occur and at quite high frequencies. For example, Sumner (1971) found 16 diploid spermatozoa in a sample of 1670 human spermatozoa $(0.96 \%)$ and Carothers \& Beatty (1975) reported $0.56 \% 2 n$ spermatozoa out of 5554 human spermatozoa. In the rabbit, Beatty \& Fechheimer (1972) found a mean incidence of $0 \cdot 31 \% 2 n$ spermatozoa for 3 strains of rabbit, a value later confirmed by Carothers \& Beatty (1975).

There is some dispute about the fertilizing ability of diploid spermatozoa. Schindler \& Mikamo (1970) agreed with Bomsel-Helmreich (1965) that there was no evidence that diploid mammalian spermatozoa were capable of successful fertilization. In man, however, it has been estimated that diploid spermatozoa account for $30 \%$ of triploid conceptions (Beatty, 1974). We have shown that diploid spermatozoa are indeed capable of fertilization in the mouse, but whether they contribute to in-vivo levels of triploidy cannot be determined from these experiments. Krzanowska (1974) has presented evidence that $2 n$ spermatozoa may be filtered out at the uterotubal junction in the mouse.

The incidence of aneuploidy, unlike that of triploidy, did not vary with PMSG dose, and the overall levels in the present work were $1.9 \%$ in vitro and $0.7 \%$ in vivo. Comparable studies by Donahue (1972a) and Kaufman (1973) of first-cleavage mouse embryos gave $1.8 \%$ and $8.3 \%$ aneuploidy, respectively. Donahue dismissed monosomic counts as artefactual due to fixation or spreading, while Kaufman included counts of $<37$ chromosomes in his estimate. In the present study, hypodiploid counts were only considered to be 'confident' if the spreads showed no irregularities. The results of the present work are in close agreement with those of Donahue and suggest that his aneuploid counts may not have been artefactual. Since additional chromosomes cannot be produced artefactually, the fact that we found no significant difference between the numbers of monosomic and trisomic counts indicates that the monosomic data are reliable.

Because polyploidy is a frequent source of developmental failure in man (Carr, 1971), the present results, albeit in mice and in vitro, suggest that hormone treatment of women and perhaps even normal fluctuations of FSH might well increase the incidence of chromosomal anomalies in human embryos.

We thank Dr C. E. Ford for his continued interest in this work and Linda Drury and Gillian Haynes for expert technical assistance. One of us (I.M.), a visiting worker at the Clinical Research Centre, is a member of the M.R.C. External Scientific Staff.

\section{References}

Austin, C.R. (1961) The Mammalian Egg. Blackwell Scientific Publications, Oxford.
BEATtY, R.A. (1970) The genetics of the mammalian gamete. Biol. Rev. 45, 73-119. 
BeATTY, R.A. (1974) Genetic aspects of spermatozoa. In Physiology and Genetics of Reproduction, pp. 183196. Eds. E. M. Coutinho \& F. Fuchs. Plenum Press, New York.

BeAtTy, R.A. \& FechHeimer, N.S. (1972) Diploid spermatozoa in rabbit semen and their experimental separation from haploid spermatozoa. Biol. Reprod. 7, 267-277.

Beaumont, H.M. \& Smith, F.A. (1975) Embryonic mortality during the pre- and post-implantation periods of pregnancy in mature mice after superovulation. J. Reprod. Fert. 45, 437-448.

BomSEL-HeLMREICH, O. (1965) Heteroploidy and embryonic death. In Pre-implantation Stages of Pregnancy, pp. 246-269. Eds G. E. W. Wolstenholme and M. O'Connor. J. \& A. Churchill, London.

Boué, J.G. \& BouÉ, A. (1973) Increased frequency of chromosomal anomalies in abortions after induced ovulation. Lancet i, 679.

BRADEN, A.W.H. (1957) Variation between strains in the incidence of various abnormalities of egg maturation and fertilization in the mouse. $J$. Genet. $55,476-486$.

Carothers, A.D. \& Beatty, R.A. (1975) The recognition and incidence of haploid and polyploid spermatozoa in man, rabbit and mouse. J. Reprod. Fert. 44, 487-500.

CARR, D.H. (1971) Chromosome studies in selected spontaneous abortions. Polyploidy in man. J. med. Genet. 8, 164-174.

Conrad, K., Buckley, J. \& Stambaugh, R. (1971) Studies on the nature of the block to polyspermy in rabbit ova. J. Reprod. Fert. 27, 133-135.

Cox, D.R. (1970) Analysis of Binary Data. Methuen Monograph, London.

DonaHuE, R.P. (1972a) Cytogenetic analysis of the first cleavage division in mouse embryos. Proc. natn. Acad. Sci. U.S.A. 69, 74-77.

Donahue, R.P. (1972b) Fertilization of the mouse oocyte: sequence and timing of nuclear progression to the two-cell stage. J. exp. Zool. 180, 305-318.

ElbliNG, L. (1973) Does gonadotrophin-induced ovulation in mice cause malformations in the offspring. Nature, Lond. 246, 37-39.

FechHeIMER, N.S. \& BeATTY, R.A. (1974) Chromosomal abnormalities and sex ratio in rabbit blastocysts. J. Reprod. Fert. 37, 331-341.

FRASER, L.R. \& DANDEXaR, P. (1975) The relationship between zona digestion and cortical granule disappearance in rabbit eggs inseminated in vitro. Biol. Reprod. 13, 123-125.

Fraser, L.R. \& DRURY, L.M. (1975) The relationship between sperm concentration and fertilization in vitro of mouse eggs. Biol. Reprod. 13, 513-518.
Fraser, L.R., Zanellotti, H.M., Paton, G.R. \& DRURY, L.M. (1976) Increased incidence of triploidy in embryos derived from mouse eggs fertilised in vitro. Nature, Lond. 260, 39-40.

Fujimoto, S., Pahlavan, N. \& Dukelow, W.R. (1974) Chromosome abnormalities in rabbit preimplantation blastocysts induced by superovulation. $J$. Reprod. Fert. 40, 177-181.

Fujimoto, S., Passantino, T.J. \& Koenczoel, I. (1975) A preliminary note on chromosome abnormalities in intratubal rabbit embryos. Proc. Japan. Acad. 51, $51-55$.

Katzberg, A.A. \& Hendrickx, A.G. (1966) Gonadotrophin-induced anomalies of the zona pellucida of the baboon ovum. Science, N.Y. 151, 1225-1226.

Kaufman, M.H. (1973) Analysis of the first cleavage division to determine the sex-ratio and incidence of chromosome anomalies at conception in the mouse. J. Reprod. Fert. 35, 67-72.

Krzanowska, H. (1974) The passage of abnormal spermatozoa through the uterotubal junction of the mouse. J. Reprod. Fert. 38, 81-90.

McGaughey, R.W. \& Chang, M.C. (1971) Chromosomes at prometaphase and metaphase of the first cleavage in mouse and hamster eggs. J. exp.Zool.177, $31-40$.

Schindler, A.M. \& Mikamo, K. (1970) Triploidy in man. Cytogenetics 9, 116-130.

ShaVer, E.L. (1970) The chromosome complement of blastocysts from rabbits injected with various doses of HCG before ovulation. J. Reprod. Fert. 23, 335337.

Smith, C.M. \& Chrisman, C.L. (1975) Failure of exogenous gonadotrophin controlled ovulation to cause digit abnormalities in mice. Nature, Lond. 253, 631-632.

SPINDle, A.I., \& Goldstein, L.S. (1975) Induced ovulation in mature mice and developmental capacity of the embryos in vitro. J. Reprod. Fert. 44, 113-116.

Stolla, R. \& Gropp, A. (1974) Variation of the DNA content of morphologically normal and abnormal spermatozoa in mice susceptible to irregular meiotic segregation. J. Reprod. Fert. 38, 335-346.

SUMNER, A.T. (1971) Frequency of polyploid spermatozoa in man. Nature, Lond. 231, 49.

TAKagi, N. \& SASAKI, M. (1976) Digynic triploidy after superovulation in mice. Nature, Lond. 264, 278-281.

TARKowskI, A.K. (1966) An air-drying method for chromosome preparations from mouse eggs. Cytogenetics 5, 394-400.

WróbleWSKA, J. (1971) Developmental anomaly in the mouse associated with triploidy. Cytogenetics 10, 199-207.

Received 9 November 1976 